\title{
Fault Diagnosis for Wireless Sensor by Twin Support Vector Machine
}

\author{
Mingli Ding, Dongmei Yang, and Xiaobing Li \\ Department of Automatic Test and Control, Harbin Institute of Technology, Harbin, Heilongjiang 150001, China \\ Correspondence should be addressed to Mingli Ding; mmingliding@126.com
}

Received 17 January 2013; Revised 20 March 2013; Accepted 7 April 2013

Academic Editor: Saeed Balochian

Copyright (c) 2013 Mingli Ding et al. This is an open access article distributed under the Creative Commons Attribution License, which permits unrestricted use, distribution, and reproduction in any medium, provided the original work is properly cited.

\begin{abstract}
Various data mining techniques have been applied to fault diagnosis for wireless sensor because of the advantage of discovering useful knowledge from large data sets. In order to improve the diagnosis accuracy of wireless sensor, a novel fault diagnosis for wireless sensor technology by twin support vector machine (TSVM) is proposed in the paper. Twin SVM is a binary classifier that performs classification by using two nonparallel hyperplanes instead of the single hyperplane used in the classical SVM. However, the parameter setting in the TSVM training procedure significantly influences the classification accuracy. Thus, this study introduces PSO as an optimization technique to simultaneously optimize the TSVM training parameter. The experimental results indicate that the diagnosis results for wireless sensor of twin support vector machine are better than those of SVM, ANN.
\end{abstract}

\section{Introduction}

In the past years, various data mining techniques including artificial neural networks have been applied to fault diagnosis for wireless sensor because they have the advantages of discovering useful knowledge from large data sets [1-5]. Though fault diagnosis for wireless sensor based on artificial neural networks can show encouraging results, there are also many problems that need to be solved, such as local optimization and overfitting in the artificial neural networks [6-11]. Support vector machine (SVM), based on structure risk minimization principle can use nonlinear mapping to transform an input space to a high-dimension space based on an internal integral function and then looks for a nonlinear relationship between inputs and outputs in that space [1214]. SVM can find global optimum solutions for problems with small training samples, high dimensions, nonlinear $[15,16]$. Twin SVM is a binary classifier that performs classification by using two nonparallel hyperplanes instead of the single hyperplane used in the classical SVM. However, the choice of the training parameters has a heavy impact on the classification accuracy of twin support vector machine. Particle swarm optimization is an evolutionary computation technique, which is inspired by social behavior among individuals. Thus, particle swarm optimization is used to optimize the TSVM parameters.

In the study, a novel classification method by twin support vector machine (PSO-TSVM) is proposed to fault diagnosis for wireless sensor, where particle swarm optimization is to find the optimal settings of parameters of SVM. Then, we collect 260 samples to study the diagnosis performance of twin support vector machine classifier, where 170 of them are used to train the diagnosis model of twin support vector machine classifier, and others are used to test the diagnosis performance of twin support vector machine classifier. The experimental results indicate that the diagnosis results for wireless sensor of twin support vector machine are better than those of SVM, ANN.

\section{Twin Support Vector Machine}

Based the Karush-Kuhn-Tucker theorem of optimization theory $[17,18]$, the nonlinear decision function is:

$$
f(x)=\operatorname{sign}\left(\sum_{i=1}^{N} \alpha_{i} y_{i} K\left(x_{i}, x_{j}\right)+b\right)
$$


The most commonly used kernel function is the radial basis function (RBF) kernel, which can be reproduced as follows:

$$
K\left(x_{i}, x_{j}\right)=\exp \left(\frac{-\left\|x_{i}-x_{j}\right\|}{2 \sigma^{2}}\right),
$$

where $\sigma$ is a positive real number.

The nonlinear TWSVM seeks two nonparallel hyperplane in $R^{n}$ :

$$
\begin{aligned}
& w_{1}^{\prime} \cdot \phi(x)+b_{1}=0, \\
& w_{2}^{\prime} \cdot \phi(x)+b_{2}=0 .
\end{aligned}
$$

For finding the hyperplanes, it is required to get the solutions to the primal problems.

Minimize

$$
U\left(w_{1}, \xi_{2}\right)=\frac{1}{2}\left\|A w_{1}+e_{1} b_{1}\right\|^{2}+c_{1} e_{2}^{\prime} \xi_{2}
$$

subject to

$$
\begin{gathered}
-\left(B w_{1}+e_{2} b_{1}\right)+\xi_{2} \geq e_{2}, \\
\xi_{2} \geq 0 \\
c_{1}>0 .
\end{gathered}
$$

And minimize

$$
U^{\prime}\left(w_{2}, \xi_{1}\right)=\frac{1}{2}\left\|B w_{2}+e_{2} b_{2}\right\|^{2}+c_{2} e_{1}^{\prime} \xi_{1}
$$

subject to

$$
\begin{gathered}
\left(A w_{2}+e_{1} b_{2}\right)+\xi_{1} \geq e_{1}, \\
\xi_{1} \geq 0 \\
c_{2}>0,
\end{gathered}
$$

where $c_{1}, c_{2}$ are the punishment parameters and $e_{1}, e_{2}$ are vectors of ones of appropriate dimensions.

\section{Parameters Optimization of TSVM by PSO}

Particle swarm optimization is an evolutionary computation technique, which is inspired by social behavior among individuals. Each particle moves in the direction of its previously best position and its best global position during each generation [19-21]. Thus, particle swarm optimization is used to optimize the TSVM parameters.

In the study, we use the RBF kernel function for the TSVM classifier because the RBF kernel function can analyze higher-dimensional data, and TSVM with RBF kernel function only has two parameters, $C$ and $\sigma$ determined. Therefore, the particle is comprised of two parts, $C$ and $\sigma$, when the RBF kernel is selected. The process of optimizing the TSVM parameters with PSO can be summarized as follows.

Step 1. Randomly generate initial population, initial particle and initial velocity.

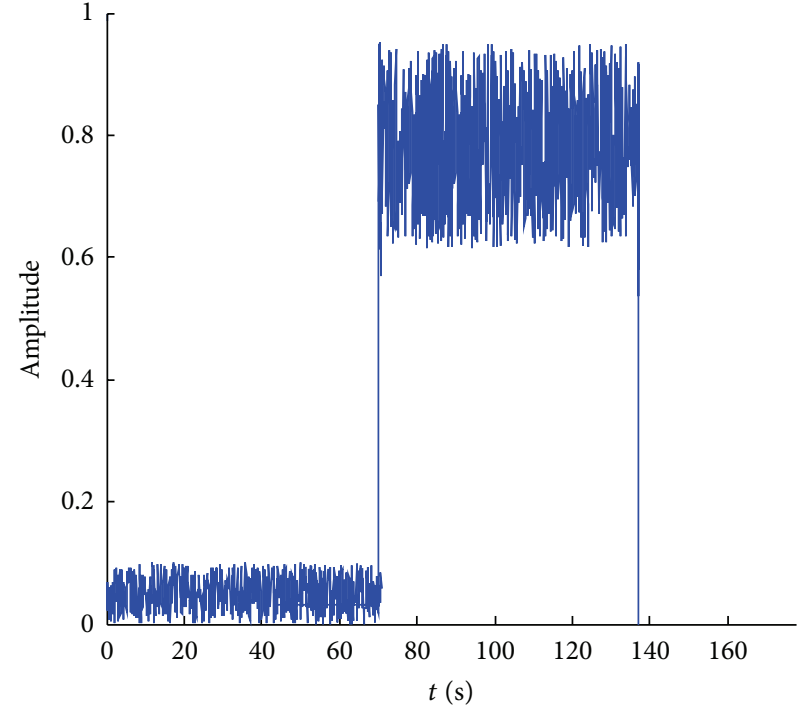

FIgURE 1: The output signal of shock failure.

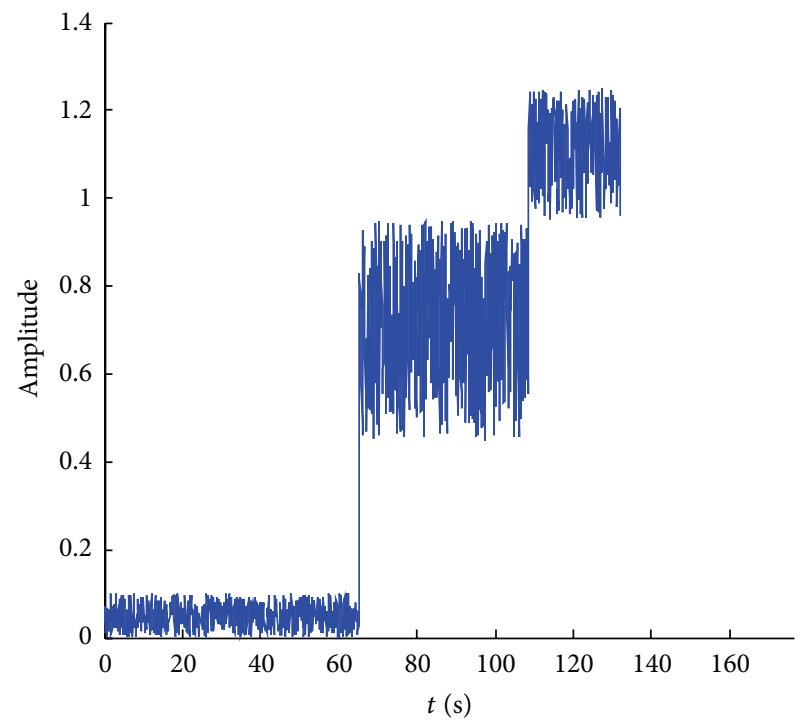

FIgURE 2: The output signal of biasing failure.

Step 2. Set the learning parameters $c_{1}$ and $c_{2}$, the inertia weight $\omega$, and the maximum number of iterations.

Step 3. Fitness evaluation: the fitness function is defined as the following formula:

$$
\text { Fitness }=\left(1-\frac{T}{F+T}\right) \times 100 \% \text {, }
$$

where $T$ denotes the correct classification and $F$ denotes the false classification.

Step 4. Update velocity and position of the particle.

Step 5. If maximum iterations predefined are met, the program is stopped. Otherwise, go to Step 3. 


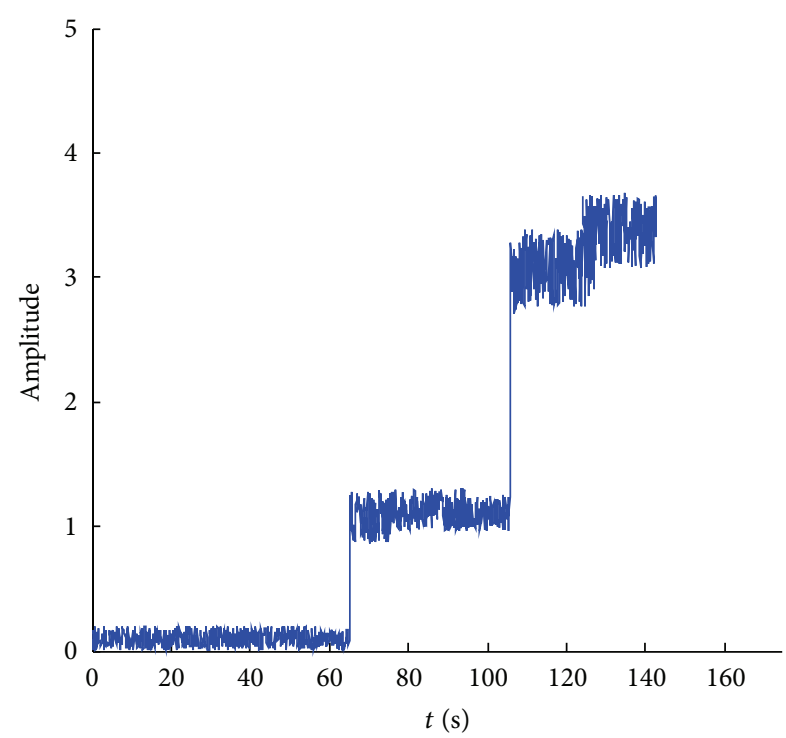

FIgURE 3: The output signal of short-circuit failure.

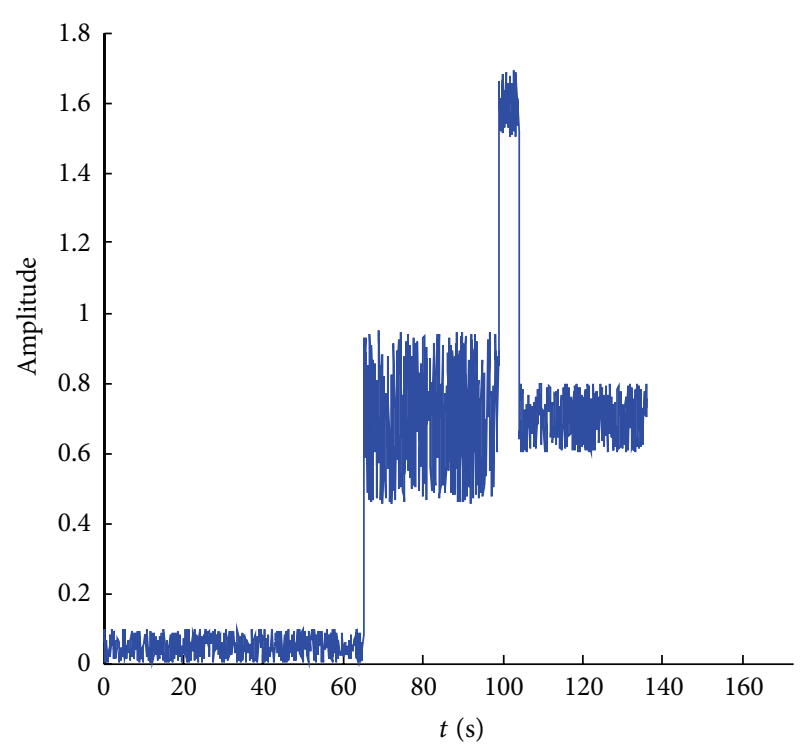

FIgURE 4: The output signal of shifting failure.

\section{Experimental Study for Fault Diagnosis of Wireless Sensor}

In the study, the four fault types of wireless sensor including shock, biasing, short circuit, and shifting are applied to test the diagnosis ability of TSVM compared with other diagnostic methods. The normal data belongs to class 1 , shock belongs to class 2 , biasing belongs to class 3 , short circuit belongs to class 4 , and shifting belongs to class 5 . The typical output signals of the above four fault types of wireless sensor can be described in Figures 1, 2, 3, and 4, respectively.

The values of the features and the corresponding state types of wireless sensor are used to train twin support vector machine classifier. In the study, we collect 260 samples to

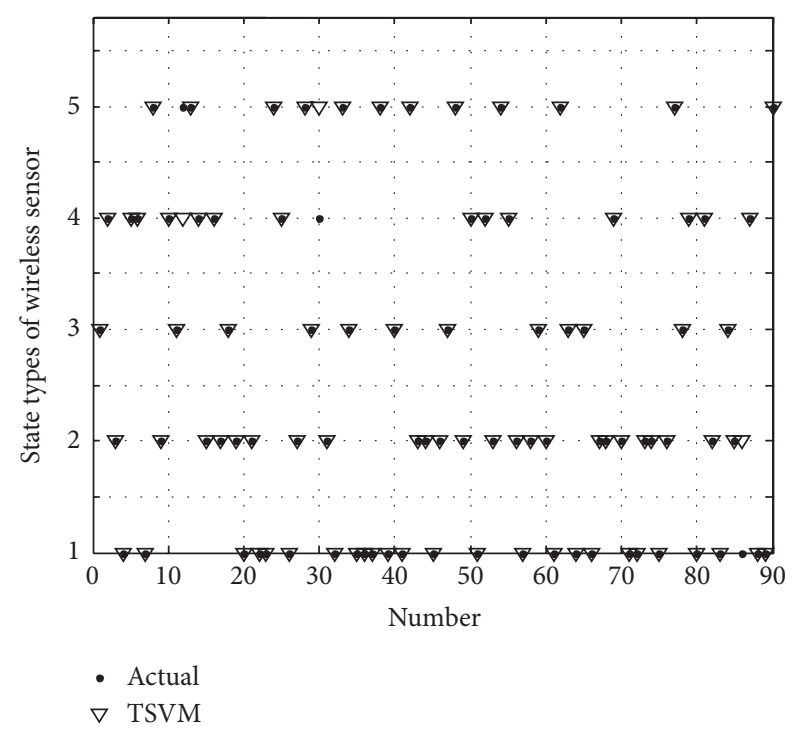

FIGURE 5: The diagnosis results of twin support vector machine.

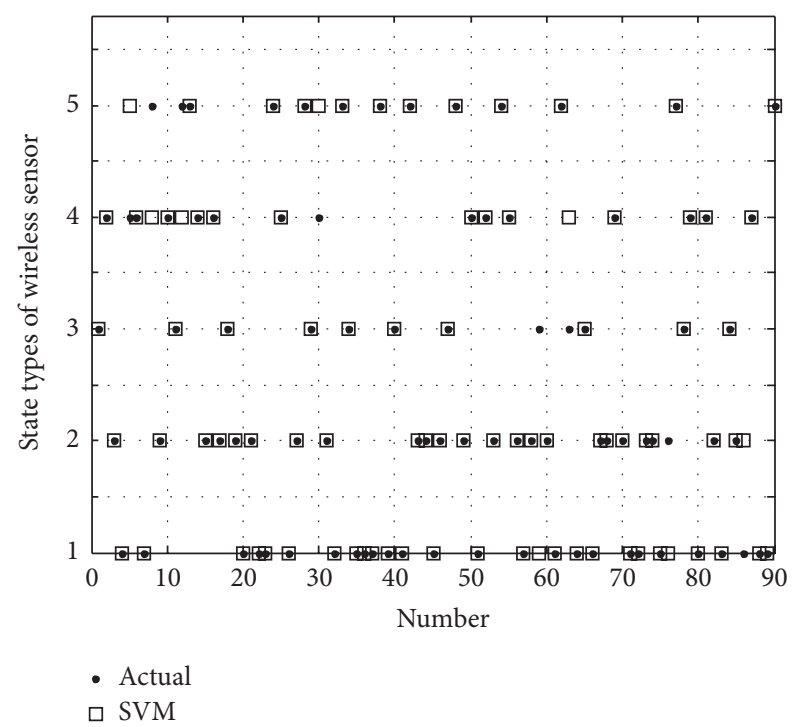

FIGURE 6: The diagnosis results of support vector machine.

study the diagnosis performance of twin support vector machine classifier, where 170 of them are used to train the diagnosis model of twin support vector machine classifier, and others are used to test the diagnosis performance of twin support vector machine classifier. Some of the experimental data are given in Table 1.

Figure 5 gives the diagnosis results of twin support vector machine, state types of wireless sensor including normal state, shock, biasing, short circuit, and shifting are given in Figure 5, which are denoted as $1 \sim 5$, respectively; Figure 6 gives the diagnosis results of the support vector machine; Figure 7 gives the diagnosis results of artificial neural network. The number of incorrect diagnosis of TSVM, SVM, and ANN is 96.7, 91.1, 83.3, respectively. The comparison of the diagnosis results for wireless sensor among TSVM, SVM, 
TABLE 1: The experimental data.

\begin{tabular}{|c|c|c|c|c|c|c|c|c|c|c|}
\hline$X_{1}$ & $X_{2}$ & $X_{3}$ & $X_{4}$ & $X_{5}$ & $X_{6}$ & $X_{7}$ & $X_{8}$ & $X_{9}$ & $X_{10}$ & Fault type \\
\hline 0.1461 & 0.1219 & 0.7039 & 1.754 & 0.7042 & 0.7212 & 0.6884 & 0.7134 & 0.6879 & 0.6333 & \multirow{8}{*}{ Shock } \\
\hline 0.1457 & 0.1227 & 0.7033 & 1.605 & 0.7122 & 0.6991 & 0.7017 & 0.7121 & 0.6671 & 0.6748 & \\
\hline 0.1462 & 0.1225 & 0.7052 & 1.669 & 0.7001 & 0.712 & 0.6657 & 0.7145 & 0.67 & 0.6501 & \\
\hline 0.1463 & 0.1226 & 0.7044 & 1.590 & 0.7183 & 0.7056 & 0.6775 & 0.7043 & 0.681 & 0.6630 & \\
\hline$\vdots$ & $\vdots$ & $\vdots$ & $\vdots$ & $\vdots$ & $\vdots$ & $\vdots$ & $\vdots$ & $\vdots$ & $\vdots$ & \\
\hline 0.1458 & 0.1221 & 0.6988 & 1.609 & 0.7211 & 0.7255 & 0.7011 & 0.7126 & 0.7028 & 0.684 & \\
\hline 0.1461 & 0.1228 & 0.7052 & 1.603 & 0.6847 & 0.6939 & 0.6507 & 0.6802 & 0.6613 & 0.6217 & \\
\hline 0.1460 & 0.1221 & 0.7045 & 1.713 & 0.7137 & 0.6977 & 0.6670 & 0.6930 & 0.6741 & 0.643 & \\
\hline 0.1462 & 0.1223 & 0.6997 & 1.119 & 1.149 & 1.164 & 1.164 & 1.148 & 1.18 & 1.161 & \multirow{7}{*}{ Biasing } \\
\hline 0.1460 & 0.1222 & 0.7027 & 1.137 & 1.153 & 1.107 & 1.149 & 1.159 & 1.166 & 1.179 & \\
\hline 0.1458 & 0.1221 & 0.6988 & 1.126 & 1.137 & 1.1 & 1.172 & 1.146 & 1.172 & 1.181 & \\
\hline 0.1459 & 0.1224 & 0.7051 & 1.153 & 1.164 & 1.124 & 1.171 & 1.164 & 1.161 & 1.163 & \\
\hline : & $\vdots$ & $\vdots$ & $\vdots$ & $\vdots$ & $\vdots$ & $\vdots$ & $\vdots$ & $\vdots$ & $\vdots$ & \\
\hline 0.1461 & 0.1228 & 0.7052 & 1.201 & 1.357 & 1.035 & 1.192 & 1.169 & 1.161 & 1.159 & \\
\hline 0.1460 & 0.1221 & 0.7045 & 1.163 & 1.167 & 1.122 & 1.153 & 1.144 & 1.172 & 1.169 & \\
\hline 0.1459 & 0.1224 & 0.7051 & 0.1853 & 0.0038 & 0.0053 & 0.001 & 0.00082 & 0.0126 & 0.0086 & \multirow{7}{*}{ Short circuit } \\
\hline 0.1462 & 0.1225 & 0.7052 & 0.1752 & 0.0016 & 0.0031 & 0.0012 & 0.0012 & 0.0115 & 0.0064 & \\
\hline 0.1460 & 0.1221 & 0.7045 & 0.1755 & 0.0003 & 0.0055 & 0.0017 & 0.0009 & 0.0127 & 0.0063 & \\
\hline 0.1458 & 0.1221 & 0.6988 & 0.1849 & 0.0011 & 0.0049 & 0.0013 & 0.00115 & 0.0086 & 0.0078 & \\
\hline$\vdots$ & $\vdots$ & $\vdots$ & $\vdots$ & $\vdots$ & $\vdots$ & $\vdots$ & $\vdots$ & : & $\vdots$ & \\
\hline 0.1462 & 0.1225 & 0.7052 & 0.1752 & 0.0016 & 0.0031 & 0.0012 & 0.0012 & 0.00115 & 0.0064 & \\
\hline 0.1461 & 0.1228 & 0.7052 & 0.1855 & 0.0002 & 0.0012 & 0.001 & 0.0088 & 0.0096 & 0.0082 & \\
\hline 0.1460 & 0.1221 & 0.7045 & 3.546 & 3.786 & 4.046 & 4.183 & 4.492 & 4.673 & 4.942 & \multirow{7}{*}{ Shifting } \\
\hline 0.1462 & 0.1225 & 0.7052 & 3.581 & 3.812 & 4.069 & 4.156 & 4.438 & 4.619 & 4.923 & \\
\hline 0.1459 & 0.1224 & 0.7051 & 3.527 & 3.846 & 4.057 & 4.191 & 4.493 & 4.615 & 4.879 & \\
\hline 0.1460 & 0.1222 & 0.7027 & 3.588 & 3.819 & 4.051 & 4.168 & 4.513 & 4.692 & 4.851 & \\
\hline$\vdots$ & $\vdots$ & : & $\vdots$ & $\vdots$ & $\vdots$ & $\vdots$ & $\vdots$ & $\vdots$ & $\vdots$ & \\
\hline 0.1457 & 0.1227 & 0.7033 & 3.593 & 3.779 & 4.053 & 4.163 & 4.472 & 4.701 & 4.928 & \\
\hline 0.1458 & 0.1221 & 0.6988 & 3.649 & 3.798 & 4.055 & 4.161 & 4.485 & 4.688 & 4.899 & \\
\hline
\end{tabular}

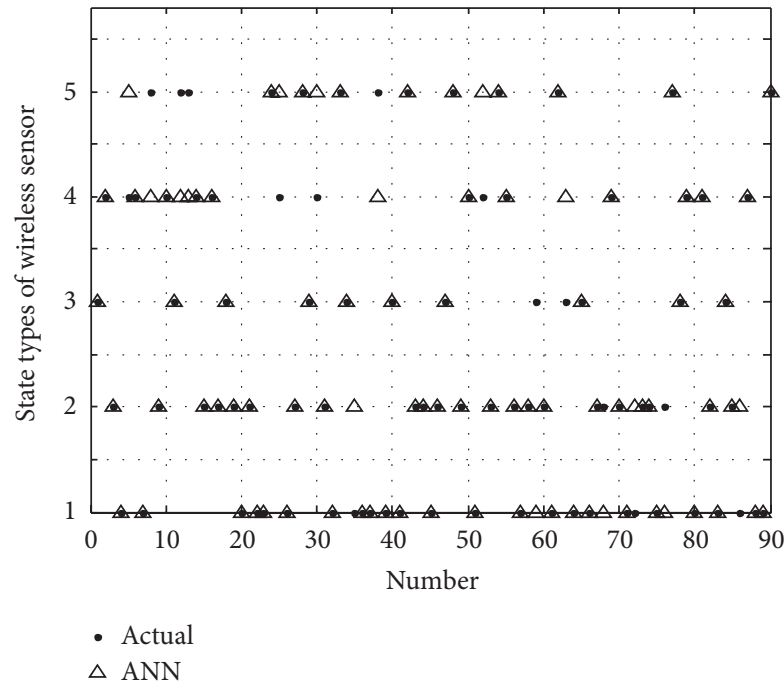

FIGURE 7: The diagnosis results of artificial neural network.
TABLE 2: The comparison of the diagnosis results for wireless sensor among the three classifiers.

\begin{tabular}{lcc}
\hline Classifier & $\begin{array}{c}\text { The number of incorrect } \\
\text { diagnosis }\end{array}$ & Diagnosis accuracy/\% \\
\hline TSVM & 3 & 96.7 \\
SVM & 8 & 91.1 \\
ANN & 15 & 83.3 \\
\hline
\end{tabular}

and ANN is given in Table 2. Then, we can conclude that the diagnosis results of twin support vector machine are better than those of SVM and ANN in the fault diagnosis of wireless sensor.

\section{Conclusion}

A novel classification method by twin support vector machine (TSVM) is proposed to fault diagnosis for wireless 
sensor in this paper, where PSO is to find the optimal settings of parameters in SVM. In the study, the four fault types of wireless sensor including shock, biasing, short circuit, and shifting are applied to test the diagnosis ability of TSVM compared with other diagnostic methods. The experimental results indicate that the diagnosis results for wireless sensor of twin support vector machine are better than those of SVM and ANN.

\section{Acknowledgments}

This work is supported by the National Natural Science Foundation of China (Grants no. 60901042 and no. 61171196).

\section{References}

[1] Z. You, X. Zhao, H. Wan, N. N. H. William, Y. Wang, and $\mathrm{M}$. Gu, "A novel fault diagnosis mechanism for wireless sensor networks," Mathematical and Computer Modelling, vol. 54, no. 1-2, pp. 330-343, 2011.

[2] H. H. Soliman, N. A. Hikal, and N. A. Sakr, "A comparative performance evaluation of intrusion detection techniques for hierarchical wireless sensor networks," Egyptian Informatics Journal, vol. 13, no. 3, pp. 225-238, 2012.

[3] K. Shao, "A novel method of transformer fault diagnosis based on extension theory and information fusion in wireless sensor networks," Energy Procedia, vol. 12, pp. 669-678, 2011.

[4] A. Díaz-Ramírez, L. A. Tafoya, J. A. Atempa, and P. MejíaAlvarez, "Wireless sensor networks and fusion information methods for forest fire detection," Procedia Technology, vol. 3, pp. 69-79, 2012.

[5] G. Li, J. He, and Y. Fu, "Group-based intrusion detection system in wireless sensor networks," Computer Communications, vol. 31, no. 18, pp. 4324-4332, 2008.

[6] F. E. Asimakopoulou, G. J. Tsekouras, I. F. Gonos, and A. Ioannis, "Stathopulos, estimation of seasonal variation of ground resistance using artificial neural networks," Electric Power Systems Research, vol. 94, pp. 113-121, 2013.

[7] C. Oz and M. C. Leu, "American sign language word recognition with a sensory glove using artificial neural networks," Engineering Applications of Artificial Intelligence, vol. 24, no. 7, pp. 12041213, 2011

[8] I. Zaier, C. Shu, T. B. M. J. Ouarda, O. Seidou, and F. Chebana, "Estimation of ice thickness on lakes using artificial neural network ensembles," Journal of Hydrology, vol. 383, no. 3-4, pp. 330-340, 2010.

[9] Q. Liu, X. Cui, M. F. Abbod, S. J. Huang, Y. Y. Han, and J. S. Shieh, "Brain death prediction based on ensembled artificial neural networks in neurosurgical intensive care unit," Journal of the Taiwan Institute of Chemical Engineers, vol. 42, no. 1, pp. 97-107, 2011.

[10] S. Khoshjavan, B. Rezai, and M. Heidary, "Evaluation of effect of coal chemical properties on coal swelling index using artificial neural networks," Expert Systems with Applications, vol. 38, no. 10, pp. 12906-12912, 2011.

[11] J. Yang, H. Singh, E. L. Hines et al., "Channel selection and classification of electroencephalogram signals: an artificial neural network and genetic algorithm-based approach," Artificial Intelligence in Medicine, vol. 55, no. 2, pp. 117-126, 2012.

[12] P. Banerjee, V. S. Singh, K. Chatttopadhyay, P. C. Chandra, and B. Singh, "Artificial neural network model as a potential alternative for groundwater salinity forecasting," Journal of Hydrology, vol. 398, no. 3-4, pp. 212-220, 2011.

[13] C. H. Wu, Y. Ken, and T. Huang, "Patent classification system using a new hybrid genetic algorithm support vector machine," Applied Soft Computing Journal, vol. 10, no. 4, pp. 1164-1177, 2010.

[14] J. Luts, G. Molenberghs, G. Verbeke, S. Van Huffel, and J. A. K. Suykens, "A mixed effects least squares support vector machine model for classification of longitudinal data," Computational Statistics \& Data Analysis, vol. 56, no. 3, pp. 611-628, 2012.

[15] D. C. Li and C. W. Liu, "A class possibility based kernel to increase classification accuracy for small data sets using support vector machines," Expert Systems with Applications, vol. 37, no. 4, pp. 3104-3110, 2010.

[16] U. B. Parikh, B. Das, and R. Maheshwari, "Fault classification technique for series compensated transmission line using support vector machine," International Journal of Electrical Power and Energy Systems, vol. 32, no. 6, pp. 629-636, 2010.

[17] H. Cevikalp, "New clustering algorithms for the support vector machine based hierarchical classification," Pattern Recognition Letters, vol. 31, no. 11, pp. 1285-1291, 2010.

[18] D. Naiyang and T. Ying-Jie, A New Data Mining MethodSupport Vector Machine, Science Press, Beijing, China, 2004.

[19] T. Navalertporn and N. V. Afzulpurkar, "Optimization of tile manufacturing process using particle swarm optimization," Swarm and Evolutionary Computation, vol. 1, no. 2, pp. 97-109, 2011.

[20] S. N. Deepa and G. Sugumaran, "Model order formulation of a multivariable discrete system using a modified particle swarm optimization approach," Swarm and Evolutionary Computation, vol. 1, no. 4, pp. 204-212, 2011.

[21] H. Wang, H. Sun, C. Li, S. Rahnamayan, and J.-S. Pan, "Diversity enhanced particle swarm optimization with neighborhood search," Information Sciences, vol. 223, pp. 119-135, 2013. 


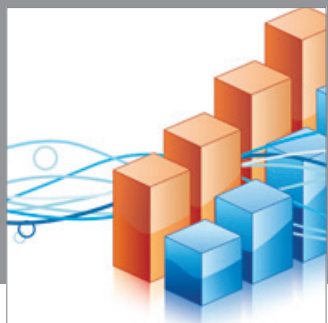

Advances in

Operations Research

mansans

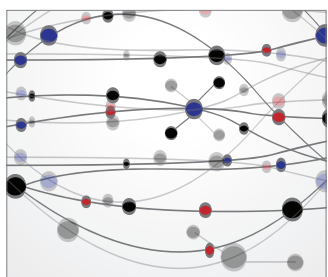

The Scientific World Journal
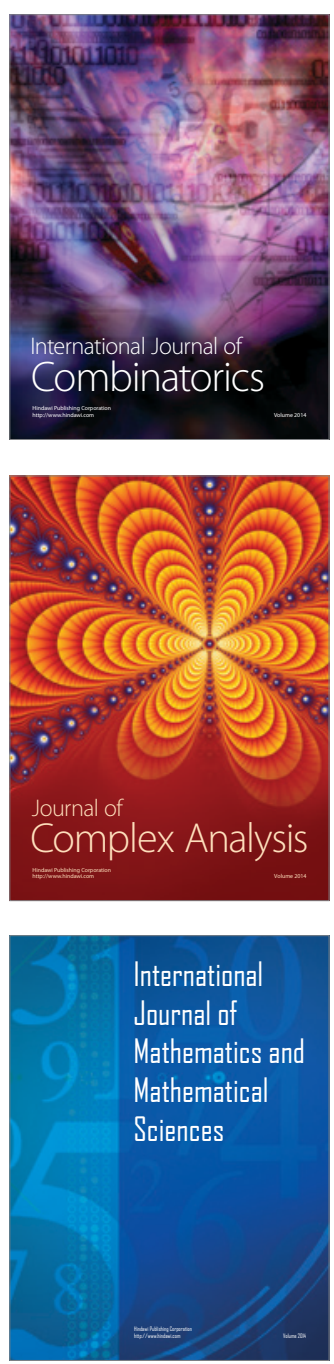
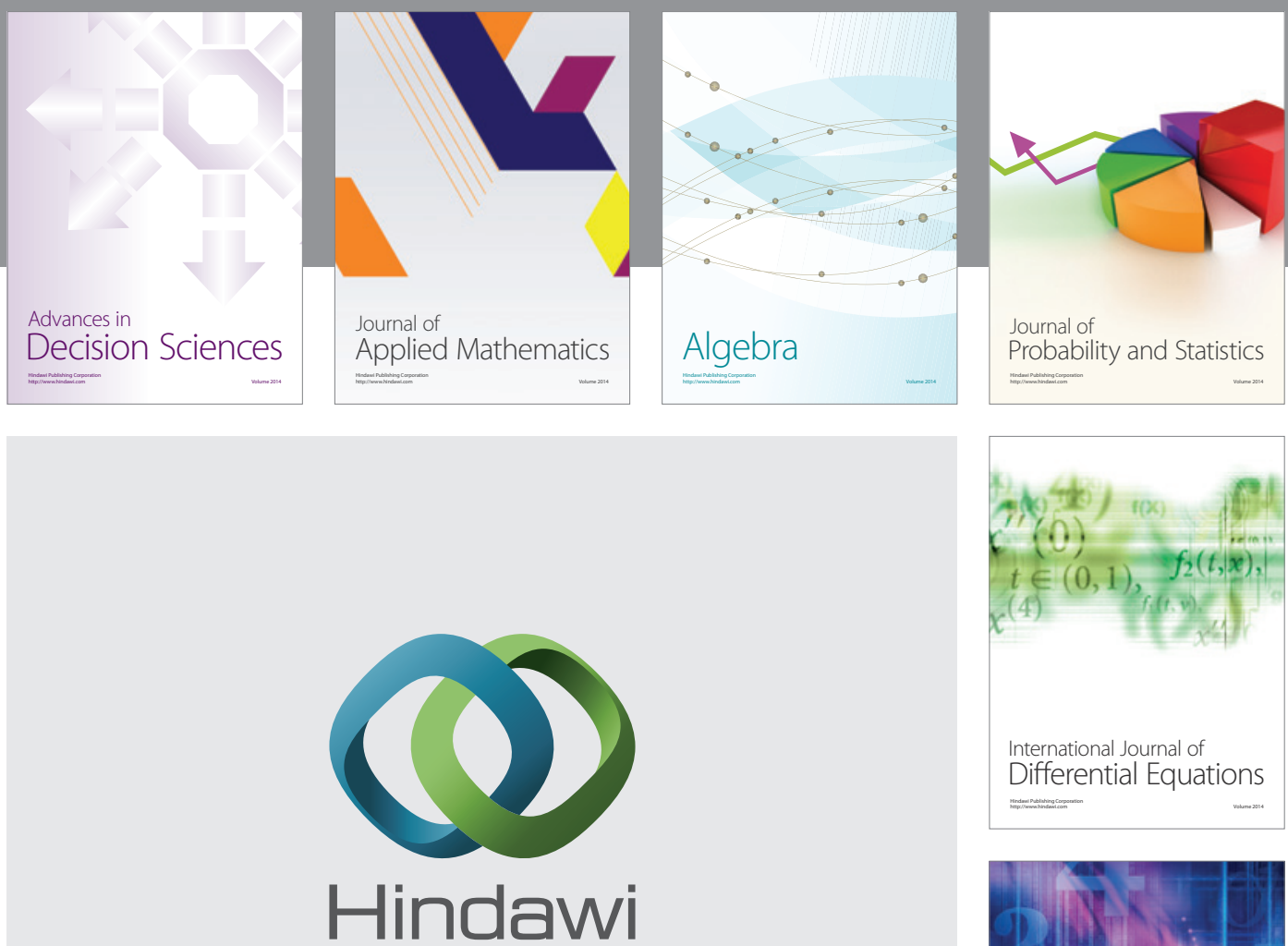

Submit your manuscripts at http://www.hindawi.com
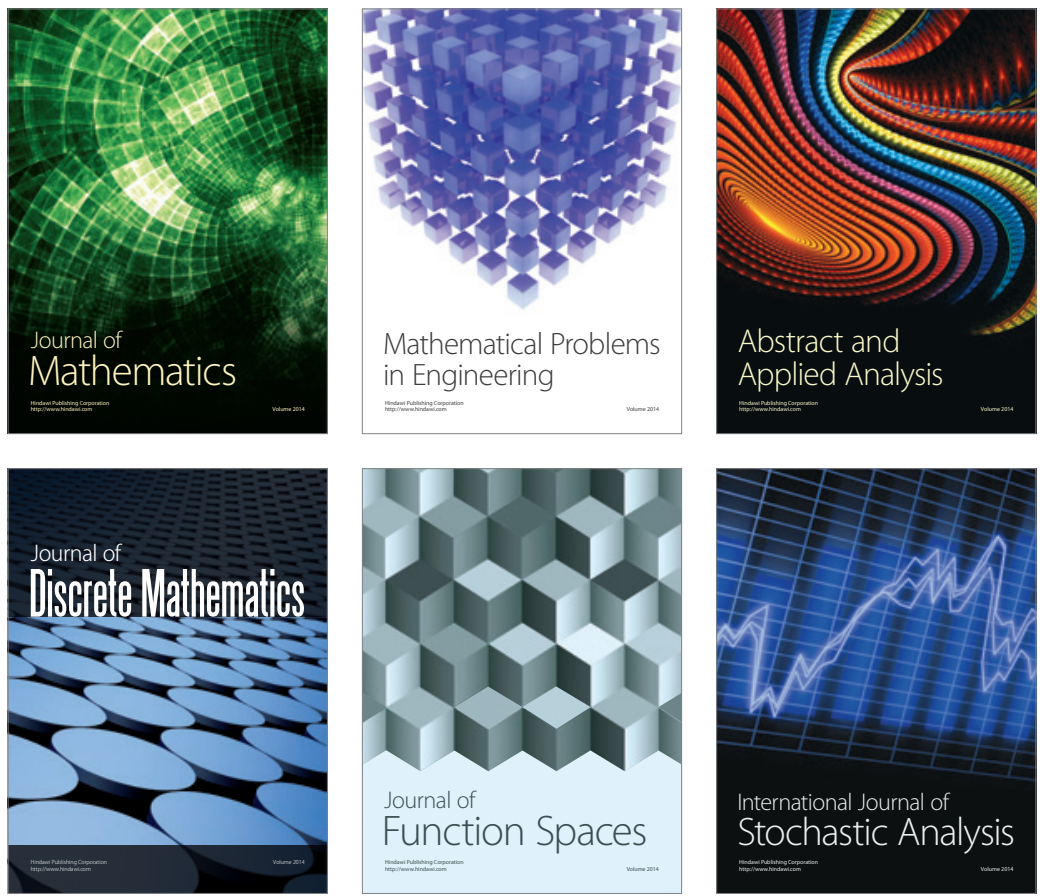

Journal of

Function Spaces

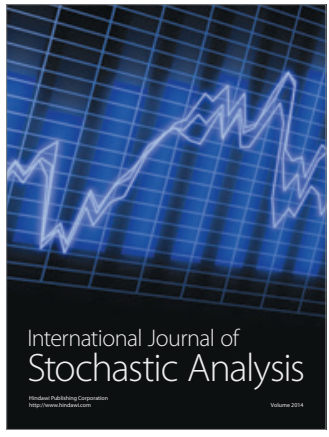

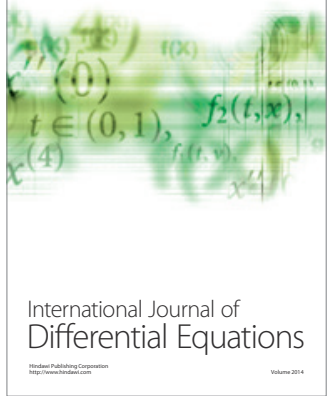
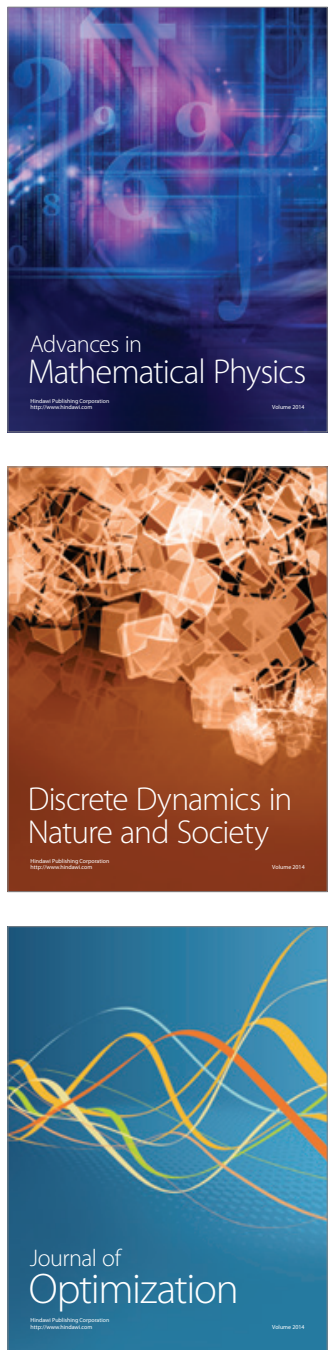